\title{
EFFICIENT SIMULATION OF RANDOM SIGNAL DETECTORS
}

\author{
Wayne T. Padgett and Douglas B. Williams \\ Georgia Institute of Technology \\ School of Electrical and Computer Engineering \\ Digital Signal Processing Laboratory \\ Atlanta, Georgia 30332-0250
}

\begin{abstract}
As the complexity of a detection algorithm increases, analytic performance evaluation becomes increasingly difficult and is often intractable. In such cases, Monte Carlo simulations can be used, but they often require an excessive amount of computation. As a means of reducing this computation, importance sampling has been applied with great success to simulations of digital communications receivers. In this paper, importance sampling strategies for the simulation of random signal detectors are presented. These strategies are shown to provide considerable computational savings over conventional Monte Carlo simulations. Additionally, simplicity and ease of use are emphasized in the development of these strategies.
\end{abstract}

\section{INTRODUCTION}

A large variety of statistical signal processing problems involve the detection of random signals. Specific applicetions range from biomedical signal analysis to spread spectrum communications to radar. For virtually all of these detection problems, Monte Carlo simulations requiring a prohibitive amount of computation are used to evaluate performance. For example, if the probability of an event, $P_{e}$ is on the order of $10^{-N}$ and a $95 \%$ confidence interval of $\left[\frac{2}{5} P_{e}, \frac{8}{5} P_{\mathrm{e}}\right]$ is required, then approximately $10^{N+1}$ independent trials are needed. The difficulty, of course, is that the events do not occur frequently enough for an accurate estimate of the event probability to be made with a small number of trials. Importance sampling, which forms a hybrid between brute force Monte Carlo simulation and analytical methods, can be used to reduce the variance of the event probability estimate so that significantly fewer trials are required. This estimate variance reduction is achieved by biasing the trials so that the event is more likely to occur but also weighting each occurrence less in the estimate of the event probability. If done properly, the estimate is still unbiased but its variance is greatly decreased for a fixed number of trials. Importance sampling has been applied with great success to simulations of digital communication receivers, but this work is the first to. apply it to random signal detection. We have derived an asymptotically optimum, yet simple, importance sampling technique for random signal detectors. This approach will be shown to yield tremendous computational savings and is directly applicable to many complex detection algorithms including nonlinear detectors, nonstationary signal detectors, detection in colored noise, and detection of signals with unknown parameters.

Many detection applications exist for which the signal of interest does not exhibit a fixed waveform but can be modelled as a stochastic process with a known second-order description. The classic example, and a very common application, is the detection of radar signals [9]. As the detector relies upon second-order descriptions of the signal and noise, the density for the $N$-dimensional observation vector $\underline{R}$ typically is assumed to be zero-mean Gaussian. Thus, the hypotheses become

$$
\begin{array}{ll}
H_{0} & : \quad \underline{R} \sim N\left(\underline{0}, \mathbf{K}_{0}\right) \\
H_{1} & : \quad \underline{R} \sim N\left(\underline{0}, \mathbf{K}_{1}\right)
\end{array}
$$

where $N(m, K)$ denotes an $N$-dimensional Gaussian probability density function with mean $\underline{m}$ and covariance matrix $K$. For most applications of interest, $K_{0}$ represents the covariance of the noise and $K_{1}$ the covariance of the signal plus noise. Because of uncertainties such as those due to unknown colored noise or nonstationary signals, the covariance matrices may contain unknown parameters that must be estimated. Therefore, quite often a fairly elaborate detector structure is necessary $[1,6,9]$. In these instances, performance analysis may not be straightforward and extensive Monte Carlo simulations are needed to evaluate quantities such as probability of false alarm $\left(P_{F}\right)$ and probability of detection $\left(P_{D}\right)$. Consequently, there is interest in reducing the required computation for these simulations. Note that because these are simulations, even if the covariance matrices are unknown to the detector, they are fixed and known for the simulations. This knowledge can be used to decrease the computation.

Many researchers have utilized importance sampling to reduce the number of trials in Monte Carlo simulations; some of the more recent results are contained in $[4,5,8]$ which also reference many of the earlier works. However, the main hindrance to using many of these results has been the analytical complexity in determining a "good" importance sampling approach. In this paper we derive an extremely simple importance sampling approach for simulating random signal detectors. Our approach is a variation on the increased variance method $[2,3]$ but differs from previous approaches in that it applies specifically to random signal detection and is a very simple result. 


\section{STRUCTURE OF THE IMPORTANCE SAMPLING STRATEGY}

The optimal random signal detector partitions the observation space into two disjoint decision regions defined by $\mathcal{Z}_{0}=\left\{\underline{R} \mid \underline{R}^{T}\left(\mathbf{K}_{0}^{-1}-\mathbf{K}_{1}^{-1}\right) \underline{R} \leq \gamma\right\}$ and $\mathcal{Z}_{1}=\mathcal{Z}_{0}^{c}$ for some threshold $\gamma$. Because the majority of the more complex random signal detectors attempt to approximate this optimum detector, they perform very similar operations and the same importance sampling strategy should be applicable.

We will now assume that we wish to estimate the probability of false alarm and note that similar operations can be performed to estimate other probabilities such as probability of detection. The standard Monte Carlo estimate is

$$
\widehat{P}_{F}=\frac{1}{M} \sum_{i=1}^{M} I_{z_{1}}\left(r_{i}\right)
$$

where the $\underline{r}_{i}$ are independent observations of the random vector $\underline{R}$ under hypothesis $H_{0}$ and $I_{z_{1}}(\cdot)$ is the indicator function defined by

$$
I_{Z_{1}}(\boldsymbol{r})= \begin{cases}1 & , \underline{r} \in \mathcal{Z}_{1} \\ 0 & , \underline{r} \in \mathcal{Z}_{0}\end{cases}
$$

Importance sampling techniques reduce the variance of this estimate by biasing the distribution of the simulated data. Instead of generating observations of the random vector $\underline{R} \sim N\left(\underline{0}, \mathbf{K}_{0}\right)$ to estimate $P_{F}$, we will generate observations of $\underline{R}^{*} \sim N\left(\underline{0}, \mathbf{K}_{*}\right)$. Thus, we will consider the alternate estimate

$$
P_{F}^{*}=\frac{1}{M} \sum_{i=1}^{M} W\left(\underline{r}_{i}^{*}\right) I_{z_{1}}\left(\underline{r}_{i}^{*}\right)
$$

where $W\left(\underline{r}_{i}^{*}\right)$ has the form of a likelihood ratio

$$
W\left(\underline{r}_{i}^{*}\right)=\frac{\frac{1}{(2 \pi)^{N / 2}\left|K_{0}\right|^{1 / 2}} \exp \left(-\frac{1}{2} \underline{r}_{i}^{* T} K_{0}^{-1} \underline{r}_{i}^{*}\right)}{\frac{1}{(2 \pi)^{N / 2}\left|K_{*}\right|^{1 / 2}} \exp \left(-\frac{1}{2} r_{i}^{* T} K_{*}^{-1} \underline{r}_{i}^{*}\right)}
$$

It is straightforward to show that $E\left[P_{F}^{*}\right]=E\left[\widehat{P}_{F}\right]=P_{F}[4]$. The problem is to determine a good choice for the biased covariance matrix $\mathbf{K}_{*}$.

Orsak and Aazhang [4] have shown that minimizing the variance of $P_{F}^{*}$ is equivalent to minimizing the quantity $\bar{W}=E_{\underline{R} \mid H_{0}}\left[W(\underline{R}) I_{z_{1}}(\underline{R})\right]$ where $E_{\underline{R} \mid H_{0}}[\cdot]$ denotes the expected value under the null hypothesis. Assuming that $\mathrm{K}_{1} \geq \mathrm{K}_{0}{ }^{1}$, which is true for many applications, the optimum value of $K$. for minimizing $\bar{W}$ in the $N$-dimensional case is approximately

$$
\begin{aligned}
\mathbf{K}_{*}= & \frac{1}{2 N}\left[\mathbf{K}_{0}+\gamma\left(\mathbf{K}_{0}^{-1}-\mathbf{K}_{1}^{-1}\right)^{-1}+\right. \\
& \left.\left(\mathbf{K}_{0}^{2}+\gamma^{2}\left(\mathbf{K}_{0}^{-1}-\mathbf{K}_{1}^{-1}\right)^{-2}\right)^{1 / 2}\right]
\end{aligned}
$$

\footnotetext{
${ }^{1}$ If $A$ and $B$ are two square matrices of the same dimensions, then $\mathbf{A} \geq \mathbf{B}$ is equivalent to $(\mathbf{A}-\mathbf{B})$ being positive semidefinite.
}

This expression appears to be a fairly complicated solution, but the limiting cases are quite simple and intuitive. Perhaps the case of most interest is the "high signal-to-noise ratio" case where $\gamma\left(\mathbf{K}_{0}^{-1}-\mathbf{K}_{1}^{-1}\right)^{-1} \gg \mathbf{K}_{0}$, and we have the very simple result $\mathbf{K}_{*} \approx(\gamma / N)\left(\mathbf{K}_{0}^{-1}-\mathbf{K}_{1}^{-1}\right)^{-1}$.

The multidimensional derivation for $\mathbf{K}_{*}$ is quite tedious and lengthy due to the multivariate calculus involved. However, it is also very similar to the 1-dimensional derivation which will be presented here.

\section{THE 1-DIMENSIONAL BIASING DISTRIBUTION}

For the 1-dimensional case, we have $\mathbf{K}_{0}=\sigma_{0}^{2}, \mathbf{K}_{1}=\sigma_{1}^{2}$, and $K_{*}=\sigma_{*}^{2}$. The objective is to minimize

$$
\begin{aligned}
\bar{W} & =\int_{z_{1}} \frac{\frac{1}{\sqrt{2 \pi} \sigma_{0}} \exp \left(-\frac{r^{2}}{2 \sigma_{0}^{2}}\right)}{\frac{1}{\sqrt{2 \pi} \sigma_{*}} \exp \left(-\frac{r^{2}}{2 \sigma_{*}^{2}}\right)} \frac{1}{\sqrt{2 \pi} \sigma_{0}} \exp \left(-\frac{r^{2}}{2 \sigma_{0}^{2}}\right) d r \\
& =\int_{\left(\frac{x^{2}}{\sigma_{0}}-\frac{r^{2}}{\sigma^{2}}\right)>\gamma} \frac{1}{\sqrt{2 \pi}} \frac{\sigma_{*}}{\sigma_{0}^{2}} \exp \left(-r^{2}\left(\frac{1}{\sigma_{0}^{2}}-\frac{1}{2 \sigma_{*}^{2}}\right)\right) d r
\end{aligned}
$$

To minimize this expression, we take the derivative with respect to $\sigma_{*}$ and set it equal to zero.

$$
\begin{aligned}
& \frac{\partial \bar{W}}{\partial \sigma_{*}}=\int \frac{1-\frac{r^{2}}{\sigma_{*}^{2}}}{\sqrt{2 \pi} \sigma_{0}^{2}} \exp \left(-r^{2}\left(\frac{1}{\sigma_{0}^{2}}-\frac{1}{2 \sigma_{*}^{2}}\right)\right) d r \\
& \left(\frac{x^{2}}{\sigma_{0}^{2}}-\frac{r^{2}}{\sigma_{1}^{2}}\right)>\gamma \\
& =0
\end{aligned}
$$

Letting

$$
x=\sqrt{\gamma\left(\frac{2}{\sigma_{0}^{2}}-\frac{1}{\sigma_{*}^{2}}\right) /\left(\frac{1}{\sigma_{0}^{2}}-\frac{1}{\sigma_{1}^{2}}\right)},
$$

and defining $Q(x)=\int_{x}^{\infty} \frac{1}{\sqrt{2 \pi}} \exp \left(-\alpha^{2} / 2\right) d \alpha$, this expression becomes

$$
\frac{1}{\gamma} \sigma_{*}^{2}\left(\frac{1}{\sigma_{0}^{2}}-\frac{1}{\sigma_{1}^{2}}\right) x^{2} Q(x)=Q(x)+\frac{1}{\sqrt{2 \pi}} x e^{-x^{2} / 2}
$$

Now for large $x, Q(x) \approx \frac{1}{\sqrt{2 \pi x}} e^{-x^{2} / 2}$; an approximation that is fairly close for $x \geq 2$ and becomes better as $x$ gets larger [7]. Using this approximation, we have

$$
\frac{1}{\gamma} \sigma_{*}^{2}\left(\frac{1}{\sigma_{0}^{2}}-\frac{1}{\sigma_{1}^{2}}\right) x^{2} Q(x)=Q(x)+x^{2} Q(x)
$$

So we need

$$
\frac{1}{\gamma} \sigma_{*}^{2}\left(\frac{1}{\sigma_{0}^{2}}-\frac{1}{\sigma_{1}^{2}}\right) x^{2}=1+x^{2}
$$

Substituting in for $x$ and simplifying we get

$$
\sigma_{*}^{4}+\left[\sigma_{0}^{2}+\gamma\left(\frac{1}{\sigma_{0}^{2}}-\frac{1}{\sigma_{1}^{2}}\right)^{-1}\right] \sigma_{*}^{2}+\frac{\sigma_{0}^{2}}{2} \gamma\left(\frac{1}{\sigma_{0}^{2}}-\frac{1}{\sigma_{1}^{2}}\right)^{-1}=0
$$


Assuming that $\sigma_{1}^{2}>\sigma_{0}^{2}$, the positive root of this quadratic equation is

$\sigma_{*}^{2}=\frac{1}{2}\left[\sigma_{0}^{2}+\gamma\left(\frac{1}{\sigma_{0}^{2}}-\frac{1}{\sigma_{1}^{2}}\right)^{-1}+\sqrt{\sigma_{0}^{4}+\gamma^{2}\left(\frac{1}{\sigma_{0}^{2}}-\frac{1}{\sigma_{1}^{2}}\right)^{-2}}\right]$

which is analogous to the multidimensional solution stated earlier.

\section{SIMULATIONS}

To evaluate the performance of the proposed importance sampling strategies, simulations were performed for 4dimensional Gaussian observation vectors $\underline{R}$. For these simulations, $K_{0}=\sigma_{n}^{2} I$ and $K_{1}=\sigma_{0}^{2} S+\sigma_{n}^{2} I$ where $S$ was a Toeplitz matrix whose first row was $\left[\begin{array}{llll}1 & 0.9 & (0.9)^{2} & (0.9)^{3}\end{array}\right]$. The detection threshold was chosen as the optimum threshold assuming equally likely hypotheses, and the parameters $\sigma^{2}$ and $\sigma_{n}^{2}$ were varied to achieve different false alarm probabilities.

To illustrate the advantages of importance sampling, "sequential" estimates of the probability of faise alarm were examined. Figure 1 shows the evolution of two estimates of the false alarm probability as trials are accumulated. The importance sampling estimate using the biasing density of equation 1 is seen to converge very rapidly. However, the simple Monte Carlo estimate varies a great deal and has not converged even after more than 4 million trials.

A reasonable measure of importance sampling performance is the importance sampling gain defined to be $M_{M C} / M_{I S}$ where $M_{M C}$ is the number of trials required to achieve a fixed variance for standard Monte Carlo simulations and MIS is the number of trials needed to achieve the same variance using importance sampling. Orsak and Aazhang [4] have shown that an estimate of the importance sampling gain is given by

$$
\frac{P_{e}^{*}\left(1-P_{e}^{*}\right)}{\bar{W}^{*}-P_{e}^{* 2}}
$$

where $P_{e}^{*}$ is the importance sampling estimate of the event probability and $\bar{W}$ is an estimate of $\bar{W}$ given by

$$
\bar{W}^{*}=\frac{1}{M} \sum_{i=1}^{M} W^{2}\left(r_{i}^{*}\right) I\left(r_{i}^{*}\right)
$$

Figure 2 shows this estimate of importance sampling gain for three cases: (1) $K$. equal to the biasing density suggested in equation $1,(2)$ the high SNR simplification $K_{.}=(\gamma / N)\left(K_{0}^{-1}-K_{1}^{-1}\right)^{-1}$, and (3) the biasing density suggested by Orsak [5] $K_{*}=K_{1}$. The biasing density proposed by Orsak is extremely simple to implement as it simply involves "swapping" the densities under hypotheses $H_{0}$ and $H_{1}$. Orsak has shown that the gain associated with this importance sampling strategy is asymptotically exponential (i.e., produces a straight line in the log-log scale used in figure 2) [5]. However, there is no guarantee that the slope of this line will not be very small as it was for this example. As is shown in figure 2, the biasing densities developed in this paper have significantly better performance than this "swapping" approach. This improvement was expected since these new biasing densities were specifically developed for random signal detectors. Note also that, because one biasing density is an asymptotic approximation to the other, their importance sampling gains become identical as the probability of false alarm decreases.

\section{CONCLUSIONS}

The proposed importance sampling strategies for random signal detectors have been shown to provide substantial computational advantages over both Monte Carlo simulations and a previous simple importance sampling strategy proposed by Orsak [5]. These new strategies are only marginally more complex than the earlier "swapping" strategy, and the only additional information that is necessary is the decision threshold $\gamma$.

\section{REFERENCES}

[1] S. Kay, "Broadband Detection of Signals with Unknown Spectra," in Proceedings of the International Conference on Acoustics, Speech, and Signal Processing, pp. 1263-1265, 1985.

[2] G. W. Lank, "Theoretical Aspects of Importance Sampling Applied to False Alarms," IEEE Thansactions on Information Theory, vol. IT-29, pp. 73-82, Jan. 1983.

[3] R. L. Mitchell, "Importance Sampling Applied to Simulation of False Alarm Statistics," IEEE Transactions on Aerospace and Electronic Systems, vol. AES-17, pp. 15-24, Jan. 1981.

[4] G. Orsak and B. Aazhang, "On the Theory of Importance Sampling Applied to the Analysis of Detection Systems," IEEE Transactions on Communications, vol. 37, pp. 332-339, Apr. 1989.

[5] G. C. Orsak, "A Note on Estimating False Alarm Rates Via Importance Sampling," IEEE Transactions on Communications, vol. 41, pp. 1275-1277, Sept. 1993.

[6] W. T. Padgett, Detection of Low Order Nonstationary Gaussian Random Processes. $\mathrm{PhD}$ thesis, Georgia Institute of Technology, Atlanta, Georgia, June 1994.

[7] H. L. Van Trees, Detection, Estimation, and Modulation Theory, Part I. New York: John Wiley and Sons, 1968.

[8] R. J. Wolfe, M. C. Jeruchim, and P. M. Hahn, "On Optimum and Suboptimum Biasing Procedures for Importance Sampling in Communication Simulation," IEEE Transactions on Communications, vol. 38, pp. 639-647, May 1990.

[9] Q. T. Zhang and S. Haykin, "Model-Based Detection," in Adaptive Radar Detection and Estimation (S. Haykin and A. Steinhardt, eds.), pp. 1-89, New York: John Wiley and Sons, 1992. 


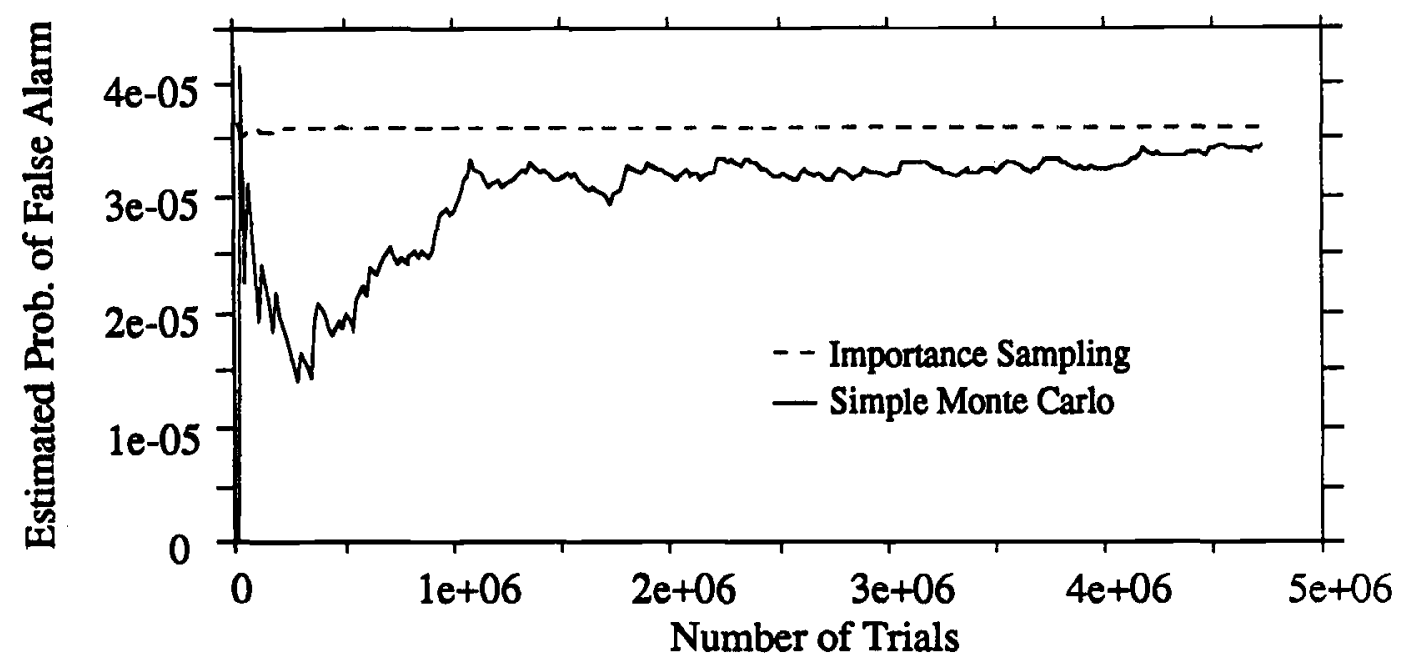

Figure 1: Plots of the estimated probability of false alarm as a function of the number of trials are shown for two simulation techniques. The solid line is the Monte Carlo estimate determined by the number of false alarms divided by the number of trials. The dashed line is the importance sampling estimate for the biasing density given in equation 1.

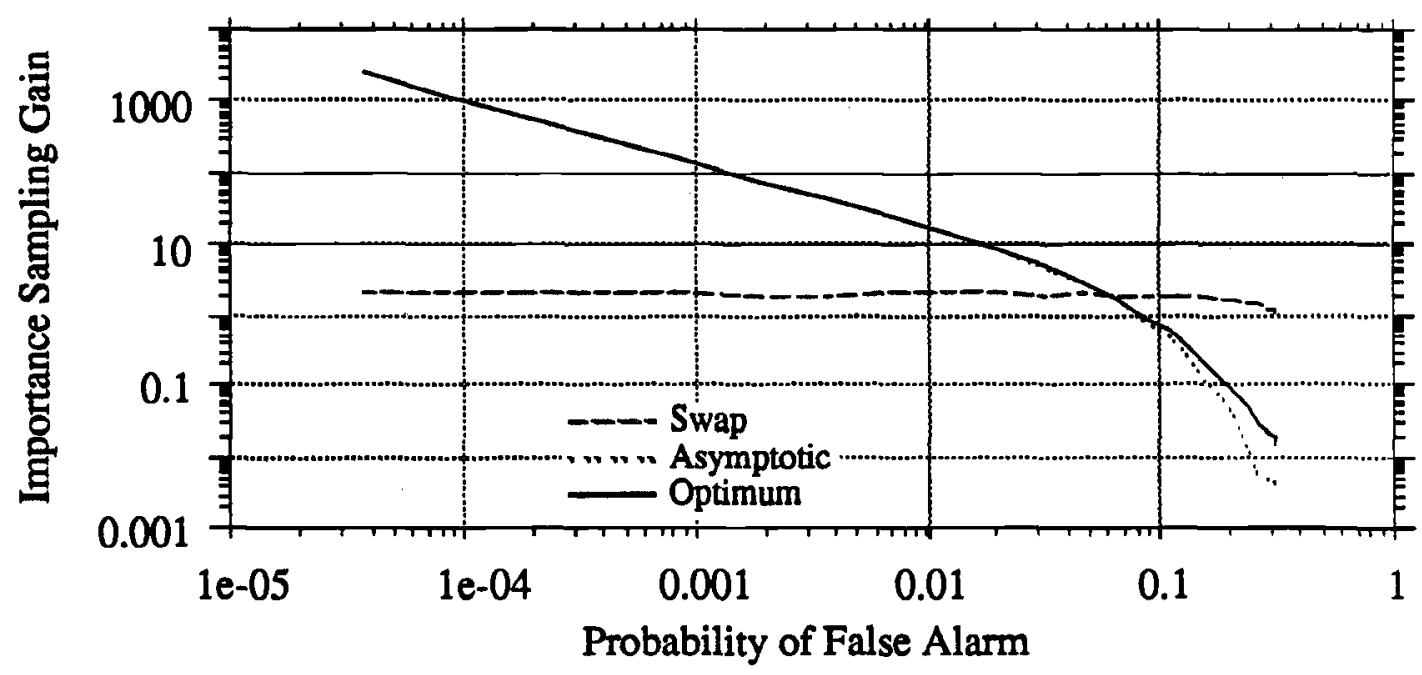

Figure 2: Plots of the estimated importance sampling gain are shown for three biasing density choices. The solid line comes from the biasing density given in equation 1, the dotted line from the high SNR approximation to that biasing density, and the dashed line from the biasing density proposed by Orsak [5]. 